\section{Asociación de labio y/o paladar hendido con variables de posición socioeconómica: un estudio de casos y controles}

\section{Association of labial and/or palatine fissure with socioeconomic variables: a case-control study}

Mauricio Escoffié-Ramírez 1

Carlo Eduardo Medina-Solís 2

América Patricia Pontigo-Loyola 3

Gladys Acuña-González 4

Juan Fernando Casanova-Rosado 5

Gabriel Eduardo Colome-Ruiz 6

1,6 Facultad de Odontología. Universidad Autónoma de Yucatán. Mérida, Yucatán., México.

2,3 Área Académica de Odontología. Instituto de Ciencias de la Salud. Universidad Autónoma del Estado de Hidalgo. Pachuca, Hidalgo, México. E-mail: cemedinas@yahoo.com

4,5 Facultad de Odontología de la Universidad Autónoma de Campeche. Campeche, Campeche, México.

\section{Resumen}

Objetivos: determinar la asociación entre varia-

Objectives: to investigate the association between socioeconomic position and the presence of nonsyndromic cleft lip and/or palate $(C L / P)$.

Methods: a case-control study with 110 cases with $C L / P$ matched by age and gender with 220 controls was carried out, the cases were selected from the "Hospital Niño DIF Hidalgo" of Pachuca, Hidalgo, Mexico. A structured questionnaire which contained socioeconomic variables was used to recollect data. Applying the principal component analysis (polycoric correlation) the socioeconomic variables were combined and were builded several socioeconomic position indicators as: socioeconomic level (house characteristics), living comfort level (house supplies), parents education (school years), social security, indigenism (native language spoken by either parent). A bivariate analysis was realized using conditional logistic regression.

Results: the highest frecuency found was the cleft lip and palate at the same time (90.9\%) either uni or bilateral. The most common defect was left cleft lip and palate (33.6\%). Cleft lip and/ or palate was associated with the living comfort level (compared with the worst quintil: 2nd $O R=0.46 ; p=0.030,3 r d$ $O R=0.39 ; p=0.015,4^{\text {th }} O R=0.30 ; p=0.002, \quad 5$ th $O R=0.27 ; p=0.001$ ), socioeconomic level (compared with the worst tercil: $\left.2^{\text {nd }} O R=0.46 ; p=0.004\right), 3 \mathrm{rd}$ $O R=0.18 ; p<0.001$, father's schooling $(O R=0.86$; $p<0.001)$, and mother's schooling $(O R=0.84$; $p<0.001)$.

Conclusions: the study demonstrates the socioeconomic inequality in oral health, observing risk for $C L / P$ according to socioeconomic position.

Key words Cleft lip, Cleft palate, Case-control study, Mexico bles indicadoras de posición socioeconómica y la presencia de labio y/o paladar hendido no sindrómico (L/PH).

Métodos: se realizó un estudio de casos y controles en el que se incluyeron 110 casos con L/PH pareados por edad y sexo con 220 controles, seleccionados de la clínica del Hospital Niño DIF de Hidalgo, México. A través de un cuestionario se recogió una serie de variables relacionadas con la posición socio-económica. Utilizando el análisis de componentes principales (correlación policórica) se combinaron las variables relacionadas entre si y se construyeron diversas variables indicadoras de posición socioeconómica; nivel socioeconómico (características de la vivienda), indice de bienestar (posesiones de bienes) enseres del hogar), escolaridad de los padres (años de estudio), seguridad social (derechohabiencia), $e$ indigenismo (hablar alguna lengua indigena por alguno de los padres). El análisis bivariado se realizó con regresión logística condicionada.

Resultados: el 90.9\% de los pacientes presentó labio + paladar hendido al mismo tiempo, ya sea uni o bilateral. El tipo de defecto mas común fue el labio y paladar hendido izquierdo (33.6\%). Resultaron asociadas a L/PH las variables: indice de bienestar (comparado con el peor quintil: 2do $O R=0.46$; $p=0.030$, 3er $O R=0.39 ; p=0.015$, 4to $O R=0.30$; $p=0.002$, 5to $O R=0.27 ; p=0.001)$, nivel socioeconómico (comparado con el mejor tercil: 2do $O R=0.46 ; p=0.004,3$ er $O R=0.18 ; p<0.001)$, escolaridad del padre $(O R=0.86 ; p<0.001)$, y escolaridad de la madre $(O R=0.84 ; p<0.001)$.

Conclusiones: este estudio demuestra la existencia de desigualdades socioeconómicas en salud bucal, observándose que los sujetos de menor posición socioeconómica presentan mayor riesgo de tener L/PH.

Palavras-chave Labio hendido, Paladar hendido, Casos y controles, México 


\section{Introducción}

Las hendiduras orofaciales, en particular la de labio y paladar hendido, constituyen un problema de salud bucodental debido a sus implicaciones e impacto en varios aspectos del desarrollo funcional y emocional a lo largo de la vida, ${ }^{1-4}$ así como económico, tanto en los hogares de pacientes como en el Sistema de Salud para su atención. Se define como una apertura alargada, que se deriva de una falta de fusión de determinadas partes durante el desarrollo embrionario. El paciente con labio y paladar hendido (LPH) presenta en mayor o menor medida alteraciones anatómicas y funcionales que interfieren con su capacidad de alimentarse, hablar u oír normalmente, a lo que se añade un compromiso estético a veces muy grave. Por su severidad y trascendencia, el labio y paladar hendido requiere una colaboración particularmente estrecha entre distintos especialistas médicos y estomatólogos. El tratamiento de estos pacientes, que comienza poco después del nacimiento, puede prolongarse hasta la vida adulta y va dirigido a: reparar la alteración morfológica, facilitar la alimentación, especialmente durante la lactancia, y permitir el desarrollo de un lenguaje normal sin pérdida de capacidad auditiva. Evitar la afectación del crecimiento maxilofacial y de la arcada dentaria superior. Para conseguir esos fines, el paciente va a precisar a lo largo de su vida numerosos procedimientos de cirugía maxilofacial, estética, otorrinolaringología, ortodoncia, periodoncia, ortopedia maxilar, prostodoncia, sesiones psicológicas. 1-4

Las fisuras pueden ser de causa genética, o desarrollarse por la acción de agentes teratógenos como: tabaco, alcohol, drogas, plaguicidas, e incluso prevenirse a través del uso de ácido fólico, entre otros agentes. Sin embargo, poco ha sido estudiado cuánto determinan los factores socioeconómicos el LPH. En este sentido, existe amplia evidencia que sugiere que la posición dentro de la estructura social es un fuerte predictor, tanto de morbilidad como de mortalidad. Además, es aceptada la existencia de una asociación entre el estado de salud y el estatus social - en general individuos de mejor nivel socioeconómico disfrutan de mejor salud. ${ }^{5}$ Además, con resultados controversiales, ya que algunos estudios no han encontrado relación alguna entre LPH con indicadores de posición socioeconómica, ${ }^{6}$ y otros, sí, han encontrado que el riesgo de presentar LPH aumenta cuando disminuye la posición socioeconómica.7-9 El enlace entre estas dos variables puede ser dado por la falta de acceso a los cuidados prenatales, una nutrición adecuada en la que estén presentes los nutrimentos necesarios para un adecuado desarrollo fetal e incluso en el acceso a mejor o peor trabajo de los padres. Por lo que el objetivo del estudio fue determinar los factores de riesgo socioeconómicos para labio y/o paladar hendido en pacientes no sindrómicos que asisten al Hospital del Niño DIF Hidalgo, en Pachuca, Hgo. México.

\section{Métodos}

Se realizó un estudio de casos y controles durante el período de Marzo - Mayo de 2007 en el Hospital del Niño, actualmente dependiente del Sistema Desarrollo Integral de la Familia de Hidago, México. El protocolo de estudio fue aprobado por el Comité de Bioética y Seguridad del Instituto de Ciencias de la Salud de la Universidad Autónoma del Estado de Hidalgo, al igual que el Hospital donde se realizó el estudio.

Fueron seleccionados como casos los pacientes consecutivos con diagnóstico de labio y/o paladar hendido que fueron inscritos en la clínica de labio y/o paladar hendido entre Marzo y Mayo de 2007, ya fuera para tratamiento quirúrgico o seguimiento, hasta completar el tamaño de muestra previamente calculado. Todos los casos fueron no sindrómicos y finalmente se incluyó un total de 110 sujetos. Los criterios de inclusión fueron: 1) Pacientes no sindrómicos con LPH de ambos sexos, inscritos para su atención en el Hospital Niño DIF Hidalgo, 2) Pacientes no sindrómicos con LPH que aceptaron ser incluidos en el estudio, 3) Pacientes no sindrómicos con LPH con queiloplastías o palatoplastías previas al momento de la exploración. Y los de exclusión: 1) Pacientes sindrómicos con LPH de ambos sexos, inscritos para su atención al Hospital Niño DIF Hidalgo, 2) Pacientes que presentaron hendidura submucosa, 3) Pacientes no sindrómicos con LPH que no aceptaron ser parte del estudio, 4) Pacientes no sindrómicos con LPH que presentaron hendiduras en lugares no descritos en la clasificación de Kernahan, 5) Pacientes quienes los tutores no pudieron aportar datos.

Cada caso fue pareado por edad y sexo con dos controles, en total 220, los cuales fueron seleccionados de entre sujetos de las listas de consultas diarias del mismo hospital, los cuales no tenían diagnóstico ni evidencia de $\mathrm{L} / \mathrm{PH}$ ni sus secuelas, lo que fue confirmado por pregunta directa e inspección clínica. Todos los sujetos invitados a participar en el estudio aceptaron ser incluidos en el mismo. Para el cálculo del tamaño de muestra se utilizó la fórmula para casos y controles: 


$$
\begin{aligned}
& n=\frac{\left[z_{1-\alpha / 2} \sqrt{(c+1) p(1-p)}+z_{1-\beta} \sqrt{c p_{1}\left(1-p_{1}\right)+p_{2}\left(1-p_{2}\right)}\right]^{2}}{c\left(p_{2}-p_{1}\right)^{2}} \\
& \text { Donde } \quad p=\frac{p_{1}+p_{2}}{2} \quad \text { y los valores } \quad z_{1-\alpha / 2} z_{1-\beta}
\end{aligned}
$$

son valores que se obtienen de la distribución normal estándar en función de la seguridad y el poder elegidos para el estudio. En el estudio se utilizó un intervalo de confianza al $95 \%$ y un poder estadístico del $80 \%$. La frecuencia de exposición en los controles fue del $11 \%$ y del $28 \%$ para los casos. 10

La variable dependiente fue la presencia/ ausencia de labio y/o paladar hendido. Las variables independientes fueron una serie de factores que determinan la posición socioeconómica (PSE). Para la conformación del primer indicador de posición socioeconómica (llamado en el estudio índice de bienestar) se incluyeron variables sobre la posesión de bienes y enseres del hogar (refrigerador, televisión, teléfono, etc). Para el segundo indicador de posición socioeconómica (llamado en el estudio índice de nivel socioeconómico) se incluyeron una serie de variables relacionadas con las características físicas del hogar (techo, paredes, piso, etc). Estas variables se combinaron utilizando correlación policórica para crear dichos índices. ${ }^{11}$ Otras variables que indicaban PSE también fueron incluidas; la institución de nacimiento, que se refiere a si los niños nacieron en instituciones públicas, de la seguridad social o privadas. La escolaridad de los padres, en esta variable se consideró el máximo grado escolar logrado. Por último el indigenismo, que hace referencia a si alguno de los padres tenía antecedentes de ser indígena, en este estudio la consideramos como si alguno de los padres hablaba algún idioma indígena.

Para la recolección de datos se utilizó un cuestionario, el cual fue previamente validado de la siguiente forma; antes de la aplicación de éste se realizó una prueba piloto en 5 casos y 5 controles para determinar si las preguntas que contenía eran entendibles para el contexto y de esta forma realizar modificaciones antes de su aplicación final. El cuestionario se dirigió a las madres/tutores de los niños. Igualmente se realizó un examen bucal para obtener la información correspondiente a la clasificación de Kernahan, ${ }^{12}$ que fue realizado por un cirujano dentista, estudiante de la maestría en ortodoncia, el cual fue previamente capacitado y estandarizado.

En el análisis estadístico, las variables fueron evaluadas en forma univariada para obtener sus porcentajes y distribuciones, de acuerdo a la escala de medición de las variables, medidas de tendencia central (media) y de dispersión (desviación estándar) para las variables cuantitavas y frecuencias y porcentaje para las cualitativas. Se realizaron pruebas estadísticas bivariadas utilizando regresión logística binaria condicionada. Debido al diseño del estudio (casos y contro-les pareado por edad y sexo), el análisis estadístico se realizó utilizando regresión logística condicional. Se calcularon odds ratio (OR) con sus intervalos de confianza al 95\% (IC95\%), así como los valores de $p$. Todos los análisis se realizaron en STATA 9.0.

\section{Resultados}

Al utilizar un diseño pareado, en ambos grupos el promedio de edad fue de $2.79 \pm 3.13$ y la distribución por sexo del $57.3 \%$ para hombres y $42.7 \%$ para mujeres.

El defecto orofacial con mayor frecuencia fue el la-bio y paladar hendido izquierdo (primario y secunda-rio) con 37 casos (33.6\%). El lado afectado con mayor frecuencia fue el izquierdo, con 42 casos (31.8\%). El labio y paladar se presentó en 100 casos $(90.9 \%)$. El defecto orofacial con mayor frecuencia en el sexo femenino y masculino fue el labio y paladar hendido izquierdo (primario y secundario) con 15 casos $(31.9 \%)$ y con 22 casos (34.9\%), respectivamente (Tabla 1). Las características socioeconómicas se muestran en la Tabla 2.

La Tabla 3 muestra los resultados obtenidos en el análisis bivariado de regresión logística condicional para LPH por posibles factores de riesgo relacionado con la posición socioeconómica.

Comparado con los del primer quintil (más pobre $)$, situarse en el segundo $(\mathrm{OR}=0.46 ; p=0.030)$, tercer $(\mathrm{OR}=0.39 ; p=0.015)$, cuarto $(\mathrm{OR}=0.30 ; p=0.002)$ y quinto $(\mathrm{OR}=0.27 ; p=0.001)$ quintil en el índice de bienestar, disminuyó el riesgo de presentar LPH. Esta asociación como vemos se comportó de forma dosis-respuesta, es decir, conforme mejor posición socioeconómica se tiene, menor riesgo de tener LPH. En cuanto al indicador de nivel socioeconómico, situarse en el 2 do $(\mathrm{OR}=0.46 ; p=0.004)$ y 3 er tercil $(\mathrm{OR}=0.18, p<0.001)$ de nivel socioeconómico disminuyó el riesgo de tener LPH. La escolaridad de la madre $(\mathrm{OR}=0.84 ; p<0.001)$; escolaridad del padre $(\mathrm{OR}=0.86, p<0.001)$ fueron estadísticamente significativos. De esta forma, por cada año que se incrementaba la escolaridad de la madre y del padre, el riesgo de tener LPH disminuyó 19\% $(1 / 0.84=1.19)$ y $16 \%(1 / 0.86=1.16)$, respectivamente. Por otro lado, el hablar alguna lengua indígena por alguno de los dos padres $(p=0.284)$; el nacimiento en institución pública ( $p=0.382$ ), no resultaron ser estadísticamente significativas en el análisis. 
Distribución por sexo del tipo de defecto observado en el estudio.

\begin{tabular}{lrrrrr}
\hline \multirow{2}{*}{ Tipo de Defecto } & \multicolumn{2}{c}{ Mujeres } & \multicolumn{2}{c}{ Hombres } & Total \\
\cline { 2 - 5 } & $\mathrm{n}$ & $\%$ & $\mathrm{n}$ & $\%$ & \\
\hline LH unilateral derecho & 0 & 0.0 & 1 & 1.6 & 1 \\
LH unilateral izquierdo & 0 & 0.0 & 0 & 0.0 & 0 \\
LPH primario izquierdo & 4 & 8.5 & 1 & 1.6 & 5 \\
LPH primario derecho & 2 & 4.3 & 1 & 1.6 & 3 \\
PH secundario (duro y blando) & 5 & 10.6 & 1 & 1.6 & 6 \\
PH secundario (blando) & 2 & 4.3 & 1 & 1.6 & 3 \\
LPH derecho (primario y secundario) & 13 & 27.7 & 18 & 28.6 & 31 \\
LPH izquierdo (primario y secundario) & 15 & 31.9 & 22 & 34.9 & 37 \\
LPH bilateral (primario y secundario) & 6 & 12.8 & 18 & 28.6 & 24 \\
Total & 47 & 42.7 & 63 & 57.3 & 110 \\
\hline
\end{tabular}

$\mathrm{LH}=$ labio hendido; $\mathrm{LPH}=$ labio y paladar hendido; $\mathrm{PH}=$ paladar hendido.

\section{Tabla 2}

Distribuciones de las variables relacionadas con la posición socioeconómica incluidas en el estudio.

\begin{tabular}{|c|c|c|c|c|}
\hline \multirow{2}{*}{ Variables } & \multicolumn{2}{|c|}{ Casos $(n=110)$} & \multicolumn{2}{|c|}{ Controles $(n=220)$} \\
\hline & $\mathrm{n}$ & $\%$ & $\mathrm{n}$ & $\%$ \\
\hline \multicolumn{5}{|l|}{ Posición socioeconómica 1 * } \\
\hline 1er quintil (más baja) & 37 & 33.6 & 34 & 15.5 \\
\hline 2do quintil & 22 & 20.0 & 44 & 20.0 \\
\hline 3er quintil & 19 & 17.3 & 42 & 19.1 \\
\hline 4 to quintil & 17 & 15.5 & 49 & 22.3 \\
\hline 5to quintil (más alta) & 15 & 13.6 & 51 & 23.2 \\
\hline \multicolumn{5}{|l|}{ Posición socioeconómica 2 † } \\
\hline 1er tercil (más baja) & 56 & 50.9 & 63 & 28.6 \\
\hline 2do tercil & 43 & 39.1 & 97 & 44.1 \\
\hline 3er tercil (más alta) & 11 & 10.1 & 60 & 27.3 \\
\hline Escolaridad de la madre (años, $\bar{X} \pm d t$ ) & \multicolumn{2}{|c|}{$8.73 \pm 2.75$} & \multicolumn{2}{|c|}{$10.49 \pm 3.61$} \\
\hline Escolaridad del padre (años, $\bar{X} \pm d t$ ) & \multicolumn{2}{|c|}{$8.25 \pm 3.69$} & \multicolumn{2}{|c|}{$10.20 \pm 3.85$} \\
\hline \multicolumn{5}{|l|}{ Indigenismo § } \\
\hline No & 100 & 90.9 & 207 & 94.1 \\
\hline Sí & 10 & 9.1 & 13 & 5.9 \\
\hline \multicolumn{5}{|l|}{ Institución de nacimiento } \\
\hline Seguridad social & 24 & 21.8 & 70 & 31.8 \\
\hline Secretaria de salud & 60 & 54.6 & 109 & 49.6 \\
\hline Particular & 26 & 23.6 & 41 & 18.6 \\
\hline
\end{tabular}

* Índice de bienestar; † Índice de NSE; § Indigenismo = se refiere si alguno de los padres habla alguna lengua indígena; $\mathrm{dt}=$ desviación típica. 
Análisis bivariado entre las variables relacionadas con la posición socioeconómica incluidas en el estudio y la presencia de labio y paladar hendido.

\begin{tabular}{|c|c|c|c|}
\hline Variables & OR & IC95\% & $p$ \\
\hline \multicolumn{4}{|l|}{ Posición socioeconómica 1 * } \\
\hline 1er quintil (más baja) & $1.00^{*}$ & & \\
\hline 2do quintil & 0.46 & $0.23-0.93$ & 0.030 \\
\hline 3er quintil & 0.39 & $0.18-0.83$ & 0.015 \\
\hline 4to quintil & 0.30 & $0.14-0.64$ & 0.002 \\
\hline 5to quintil (más alta) & 0.27 & $0.13-0.58$ & 0.001 \\
\hline \multicolumn{4}{|l|}{ Posición socioeconómica 2 † } \\
\hline 1er tercil (más baja) & $1.00^{*}$ & & \\
\hline 2do tercil & 0.46 & $0.27-0.78$ & 0.004 \\
\hline 3er tercil (más alta) & 0.18 & $0.08-0.39$ & $<0.001$ \\
\hline Escolaridad de la madre & 0.84 & $0.78-0.91$ & $<0.001$ \\
\hline Escolaridad del padre & 0.86 & $0.80-0.93$ & $<0.001$ \\
\hline \multicolumn{4}{|l|}{ Indigenismo } \\
\hline No & $1.00^{*}$ & & 0.284 \\
\hline Sí & 1.61 & $0.67-3.85$ & \\
\hline \multicolumn{4}{|l|}{ Institución de nacimiento } \\
\hline Seguridad social y particular & $1.00^{*}$ & & 0.382 \\
\hline Secretaria de salud & 1.24 & $0.77-1.97$ & \\
\hline
\end{tabular}

$\mathrm{OR}=$ odds ratio; * Índice de bienestar; † Índice de NSE; § Indigenismo = se refiere si alguno de los padres habla alguna lengua indígena.

\section{Discusión}

Este estudio encontró evidencia de asociación de labio y/o paladar hendido con la posición socioeconómica tanto a nivel individual como del hogar, incluso esta asociación mantuvo una dosisrespuesta. El LPH, por su complejidad y carga económica para su terapéutica, resulta ser una anomalía craneofacial que representa un problema de salud pública. De forma similar, existen otros defectos de nacimiento (defectos del tubo neural) que se encuentran relacionados con el LPH, y que también muestran asociación con la PSE.13-15

Según estudios realizados por Jensen et al., 16 Hagberg et al., ${ }^{17}$ Suleiman et al., 18 la mayor prevalencia de LPH y LPH bilateral fue en varones, el lado de mayor prevalencia fue el izquierdo, así como la mayoría de los pacientes con fisuras fueron del género masculino, coincidiendo con los resultados observados en el presente trabajo. Por tipo de defecto, en el presente estudio, se observó con mayor prevalencia LPH, seguido de sólo $\mathrm{PH}$ y LH, respectivamente, observándose resultados similares a estudios realizados por Pérez-Molina, ${ }^{19}$ Al Omari y Al Omari.20
Aunque las variables relacionadas con la posición socioeconómica han sido poco analizadas, cuando se estudian los defectos orofaciales, se ha observado de manera consistente que las condiciones desfavorables de la posición socioeconómica se asocia a la presencia de LPH como refieren Clark et al.,8 Puho et al., ${ }^{9}$ Carmichael et al.,21 y Van Roij et $a l .22$ En este estudio se observó que, en las variables indicadoras socioeconómicas como, índice de bienestar, nivel socioeconómico y escolaridad de la madre y el padre, siempre los mayores porcentajes fueron los de las categorías más negativas o de peor posición socioeconómica, con excesos de riesgo de hasta 5.55 (ver Tabla 3: $1 / 0.18=5.55$ ) veces comparando al mejor y al peor posicionados. Lo que sí es conclusivo es la asociación de los resultados de salud y la posición socioeconómica, donde se observa lo que se ha denominado gradiente social en salud; el cual se refiere a que las desigualdades en la distribución del estado de salud de la población están relacionadas a las desigualdades en el estatus social, 23 de esta manera, los sujetos que se encuentran peor posicionados socioeconómicamente tendrán peores resultados en salud. Esto puede ser observado en México, incluso cuando se estudian 
otros aspectos relacionados con la salud bucal.24-27

Entre las limitaciones del estudio está el potencial sesgo, que se presenta en todos los estudios de casos y controles: la introducción de sesgo de memoria, ya que no se puede reconstruir completamente la historia de exposición de manera retrospectiva, y este hecho puede ser mayor en los controles

\section{Referencias}

1. Kramer FJ, Gruber R, Fialka F, Sinikovic B, Hahn W, Schliephake H. Quality of life in school-age children with orofacial clefts and their families. J Craniofac Surg. 2009; 20: 2061-6.

2. Bernheim N, Georges M, Malevez C, De Mey A, Mansbach A. Embryology and epidemiology of cleft lip and palate. BENT. 2006; 2 (Suppl 4): 11-9.

3. Mossey PA, Little J, Munger RG, Dixon MJ, Shaw WC Cleft lip and palate. Lancet. 2009; 374 (9703): 1773-85.

4. Yu W, Serrano M, Miguel SS, Ruest LB, Svoboda KK Cleft lip and palate genetics and application in early embryological development. Indian J Plast Surg. 2009; 42 (Suppl): S35-50.

5. Laaksonen M, Rahkonen O, Martikainen P, Lahelma E. Socioeconomic Position and Self-Rated Health: The Contribution of Childhood Socioeconomic Circumstances, Adult Socioeconomic Status, and Material Resources. Am J Public Health. 2005; 95: 1403-9.

6. Kallen K. Maternal smoking and orofacial clefts. Cleft Palate Craniofac J. 1997; 34: 11-6.

7. Carmichael SL, Ma C, Shaw GM. Socioeconomic measures, orofacial clefts, and conotruncal heart defects in California. Birth Defects Res A Clin Mol Teratol. 2009; 85: 850-7.

8. Clark JD, Mossey PA, Sharp L, Little J. Socioeconomic status and orofacial clefts in Scotland, 1989 to 1998. Cleft Palate Craniofac J. 2003; 40: 481-5

9. Puho E, Metneki J, Czeizel AE. Maternal employment status and isolated orofacial clefts in Hungary. Cent Eur J Public Health. 2005; 13: 144-8.

10. Acuña-González G, Medina-Solis CE. Factores de riesgo para defectos orofaciales congénitos en el Estado de Campeche. Libro de resúmenes XIII Encuentro Nacional y IV Encuentro Iberoamericano de Investigación en Odontología. Pag. 129, Res 75. (ISBN: 970-769-013-5).

11. Kolenikov S, Angeles G. The Use of Discrete Data in Principal Component Analysis With Applications to SocioEconomic Indices. CPC/MEASURE Working paper No. WP-04-85. 2004

12. Kernahan DA. The striped Y - a symbolic classification for cleft lips and palates. Plast Reconstr Surg. 1971; 47: 46970.

13. Blanco Munoz J, Lacasana M, Borja Aburto VH, Sánchez LET, García García AM, Carrillo LL. Socioeconomic factors and the risk of anencephaly in a Mexican population: a case-control study. Public Health Rep. 2005; 120: $39-45$ que en los casos, lo cual daría como resultado la sobrestimación del efecto de los factores de riesgo. Sin embargo, podemos concluir que este estudio demuestra la existencia de desigualdades socioeconómicas en salud bucal, observándose que los sujetos de menor posición socioeconómica presentan mayor riesgo de tener $\mathrm{L} / \mathrm{PH}$.
14. Nili F, Jahangiri M. Risk factors for neural tube defects: a study at university-affiliated hospitals in Tehran. Arch Iran Med. 2006; 9: 20-5.

15. Rull RP, Ritz B, Shaw GM. Neural tube defects and maternal residential proximity to agricultural pesticide applications. Am J Epidemiol. 2006; 163: 743-53.

16. Jensen BL, Kreiborg S, Dahl E, Fogh-Andersen P. Cleft lip and palate in Denmark, 1976-1981: epidemiology, variability, and early somatic development. Cleft Palate J. 1988; 25: 258-69

17. Hagberg C, Larson O, Milerad J, Incidence of cleft lip and palate and risks of additional malformations. Cleft Palate Craniofac J. 1998; 35: 40-5.

18. Suleiman AM, Hamzah ST, Abusalab MA, Samaan KT. Prevalence of cleft lip and palate in a hospital-based population in the Sudan. Int J Paediatric Dent. 2005; 15: 185-9.

19. Pérez-Molina JJ, Alfaro-Alfaro N, Angulo-Castellanos., Prevalencia y factores de riesgo de labio y paladar hendido en dos hospitales, en la ciudad de Guadalajara, Jalisco, México. Bol Med Hosp Infant Mex. 1993; 50: 110-3.

20. Al Omari F, Al Omari IK. Cleft lip and palate in Jordan: Birth prevalence rate. Cleft Palate Craniofac J. 2004; 41: 609-12.

21. Carmichael SL, Nelson V, Shaw GM, Wasserman CR, Croen LA. Socio-economic status and risk of conotruncal heart defects and orofacial clefts. Paediatr Perinat Epidemiol. 2003; 17: 264-71.

22. Van Rooij IALM, Vermeij-Keers C, Kluijtmans LAJ, Ocké MC, Zielhuis GA, Goorhuis-Brouwer SM, van der Biezen JJ, Kuijpers-Jagtman AM, Steegers-Theunissen RP. Does the interaction between maternal folate Intake and the Methylenetetrahydrofolate Reductase polymorphisms affect the risk of cleft lip with or without cleft palate? Am J Epidemiol. 2003; 157: 583-91.

23. Kosteniuk JG, Dickinson HD. Tracing the social gradient in the health of Canadians: primary and secondary determinants. Soc Sci Med. 2003; 56: 263-76.

24. Medina-Solis CE, Maupomé G, Pelcastre-Villafuerte B, Avila-Burgos L, Vallejos-Sánchez AA, Casanova-Rosado AJ. Desigualdades socioeconómicas en salud bucal: caries dental en niños de 6 a 12 años de edad. Rev Invest Clin. 2006; 58: 296-304.

25. Villalobos-Rodelo JJ, Medina-Solís CE, Maupomé G, Vallejos-Sánchez AA, Lau-Rojo L, Ponce de León-Viedas MV. Socioeconomic and socio-demographic variables associated with oral hygiene status in Mexican schoolchildren aged 6 to 12 years. J Periodontol. 2007; 78: 816-22. 
26. Villalobos-Rodelo JJ, Medina-Solís CE, Maupomé G Pontigo-Loyola AP, Lau-Rojo L, Verdugo-Barraza L. Caries dental en escolares de una comunidad del Noroeste de México con dentición mixta, y su asociación con algunas variables clínicas, socioeconómicas y sociodemográficas. Rev Invest Clin. 2007; 59: 256-67.

Recebido em 7 de agosto de 2009

Versão final apresentada em 8 de junho de 2010

Aprovado em 21 de junho de 2010
27. Medina-Solis CE, Pérez-Núñez R, Maupomé G, CasanovaRosado JF. Edentulism among Mexicans 35 years old and older, and associated factors. Am J Public Health. 2006; 96: 1578-81. 